\title{
Pseudo differential operators with negative definite symbols of variable order
}

Walter Hoh

\section{Introduction.}

One way to represent the generator of a Markov process is given by pseudo differential operators. Above all this is due to the fact that the generator satisfies the so-called positive maximum principle. Then by a result of $\mathrm{Ph}$. Courrège [4] it is known that a linear operator $A$ : $C_{0}^{\infty}\left(\mathbb{R}^{n}\right) \longrightarrow C\left(\mathbb{R}^{n}\right)$ which satisfies the positive maximum principle, that is for any $\varphi \in C_{0}^{\infty}\left(\mathbb{R}^{n}\right)$ and $x_{0} \in \mathbb{R}^{n}$ we have

$$
\varphi\left(x_{0}\right)=\sup _{x \in \mathbb{R}^{n}} \varphi(x) \geq 0 \quad \text { implies } \quad A \varphi\left(x_{0}\right) \leq 0,
$$

has a representation as a pseudo differential operator

$$
A \varphi(x)=-p(x, D) \varphi(x)=-\int_{\mathbb{R}^{n}} e^{i(x, \xi)} p(x, \xi) \cdot \hat{\varphi}(\xi) d \xi
$$

with $\varphi \in C_{0}^{\infty}\left(\mathbb{R}^{n}\right)$. Here the symbol $p(x, \xi)$ is a function $p: \mathbb{R}^{n} \times$ $\mathbb{R}^{n} \longrightarrow \mathbb{C}$ which has the basic property that for fixed $x \in \mathbb{R}^{n}$ the function $\xi \longmapsto p(x, \xi)$ is a continuous and negative definite function. Symbols with this property we call negative definite symbols. We refer to Berg, Forst [3] for the definition and properties of negative definite 
functions. Conversely a negative definite symbol defines by (1.1) an operator satisfying the positive maximum principle. Here

$$
\hat{\varphi}=\int_{\mathbb{R}^{n}} e^{-i(x, \xi)} \varphi(x) d x
$$

denotes the Fourier transform and $\bar{d} \xi=(2 \pi)^{-n} d \xi$.

Starting with a symbol of this type there had been several attempts to construct an associated Markov process or semigroup (see Jacob [13], [14] and also [7], [8], [9]). The fundamental idea is to fix a continuous negative definite function $a^{2}: \mathbb{R}^{n} \longrightarrow \mathbb{R}$ as a reference function. Then the $x$-independent symbol $a^{2}(\xi)$ defines by (1.1) an operator $-a^{2}(D)$ and it is well-known that $-a^{2}(D)$ is the generator of the Lévy process with characteristic exponent $a^{2}(\xi)$. In [7], [8], [9] and [13], [14] assumptions on the symbol $p(x, \xi)$ are expressed in terms of the reference function $a^{2}(\xi)$. More precisely, there are upper bounds of the symbol and its derivatives with respect to $x$ up to a certain order

$$
\partial_{x}^{\beta} p(x, \xi) \leq c\left(1+a^{2}(\xi)\right)
$$

as well as a lower bound

$$
p(x, \xi) \geq \delta a^{2}(\xi), \quad|\xi| \text { large },
$$

which should be regarded as a kind of ellipticty condition. But note that (1.3) is no ellipticty condition in the usual sense since the growth of $a^{2}$ might be quite anisotropic and hence the symbol might be degenerated.

Under assumptions of this type it was shown that either the operator extends to the generator of a Feller semigroup or the corresponding martingale problem is well-posed.

The purpose of this article is to give similar results in an explicitly non-elliptic situation, that is in the case of operators of variable order. Recently several investigations were made in the case of the best known example, the generator of the so-called stable-like process which is given by the symbol

$$
p(x, \xi)=|\xi|^{\alpha(x)}
$$

or to avoid problems with differentiability

$$
p(x, \xi)=\left(1+|\xi|^{2}\right)^{\alpha(x) / 2},
$$


where $0<\alpha(x) \leq 2$. For fixed $x$ the operator $-p(x, D)$ coincides with the generator of a symmetric $\alpha(x)$-stable process, but the order varies with $x$. Note that in particular for fixed $x$ the symbol is a negative definite function.

In the one-dimensional situation Bass [1] proved well-posedness of the corresponding martingale problem under weak assumptions on $\alpha(x)$. In the higher dimensional case the process corresponding to (1.4) was constructed by Tsuchiya [29] as the solution of a stochastic differential equation. Symbols as in (1.5) are contained in the Hörmander classes $S_{\varrho, \delta}^{m}$ and were studied by the symbolic calculus of pseudo differential operators by Unterberger, Bokobza [27], [28], Unterberger [26], Višik, Eskin [31], [32] and Beauzamy [2].

In [15] Jacob and Leopold constructed a Feller semigroup generated by operators with symbols (1.5). Their approach is mainly based on the method of Jacob concerning the generation of Feller semigroups by pseudo differential operators in [13], [14], and the results of Leopold on pseudo differential operators of variable order and corresponding Sobolev spaces, [17], [18], [19]. See also Negoro [25] and Kikuchi, Negoro $[22]$ for further results concerning existence of transition densities and path behaviour of stable-like processes.

In this paper we consider a more general situation. The functions $|\xi|^{2}$ and $1+|\xi|^{2}$ in (1.4) and (1.5) are associated to a diffusion process, i.e. Brownian motion. Thus the stable-like process can be regarded as a diffusion subordinated by a subordinator given by the exponent $\alpha(x) / 2$, but the subordinator depends on $x$. Our starting point will be the generator $-a^{2}(D)$ of a Lévy process or even a generator with variable coefficients which satisfies upper and lower estimates with respect to $a^{2}(\xi)$ in the sense of $(1.2),(1.3)$. We denote this symbol by $s(x, \xi)$ and consider the symbol

$$
p(x, \xi)=s(x, \xi)^{m(x)},
$$

where $0<m(x) \leq 1$. Note that if $s(x, \xi)$ is a negative definite symbol, then $p(x, \xi)$ also is negative definite.

Negative definite symbols are in general not in Hörmander classes $S_{\varrho, \delta}^{m}$, they are even not differentiable with respect to $\xi$. But if we restrict to the situation that the associated jump type processes only have jumps of bounded size, then the corresponding negative definite functions turn out to be infinitely often differentiable and certain estimates for the derivatives hold true. In [9], see also [10], this behaviour is used as the motivation to define appropriate symbol classes $S_{\varrho}^{m, \lambda}$. More precisely, let $a^{2}: \mathbb{R}^{n} \longrightarrow \mathbb{R}$ be a continuous negative definite reference 
function as above with the property that the Lévy measure of $a^{2}$ has bounded support (see Section 2). Moreover we will assume that $a^{2}$ has a minimal growth behaviour at infinity, i.e. there are constants $r>0$ and $c>0$ such that

$$
a^{2}(\xi) \geq c|\xi|^{r}, \quad|\xi| \text { large } .
$$

It turns out to be more convenient to express estimates for the symbol in terms of the square root

$$
\lambda(\xi):=\left(1+a^{2}(\xi)\right)^{1 / 2}
$$

instead of $a^{2}$ itself. Moreover let

$$
\varrho(k):=k \wedge 2, \quad k \in \mathbb{N}_{0}
$$

Then the class $S_{\varrho}^{m, \lambda}$ of symbols of order $m \in \mathbb{R}$ is defined as the set of all $C^{\infty}$-functions $p: \mathbb{R}^{n} \times \mathbb{R}^{n} \longrightarrow \mathbb{C}$ such that

$$
\left|\partial_{\xi}^{\alpha} \partial_{x}^{\beta} p(x, \xi)\right| \leq c_{\alpha, \beta} \lambda(\xi)^{m-\varrho(|\alpha|)},
$$

where $x \in \mathbb{R}^{n}, \xi \in \mathbb{R}^{n}, \alpha, \beta \in \mathbb{N}_{0}^{n}$. Negative definite symbols that can be compared to $a^{2}(\xi)$ typically belong to $S_{\varrho}^{2, \lambda}$ (see also Section 2). Our main result is the following

Theorem 1.1. Let $a^{2}: \mathbb{R}^{n} \longrightarrow \mathbb{R}$ be a continuous negative definite function such that the Lévy measure of $a^{2}$ has bounded support and (1.7) holds. Let $s \in S_{\varrho}^{2, \lambda}$ be a real-valued negative definite symbol which is elliptic, i.e. there is a $\delta>0$ such that

$$
s(x, \xi) \geq \delta \lambda^{2}(\xi)
$$

Consider a $C^{\infty}$-function $m: \mathbb{R}^{n} \longrightarrow(0 ; 1]$ with bounded derivatives and let $M:=\sup _{x \in \mathbb{R}^{n}} m(x), \mu:=\inf _{x \in \mathbb{R}^{n}} m(x)$.

If

$$
M-\mu<\frac{1}{2} \quad \text { and } \quad \mu>0,
$$

then

$$
p(x, \xi)=s(x, \xi)^{m(x)}
$$


defines by (1.1) an operator $-p(x, D): C_{0}^{\infty}\left(\mathbb{R}^{n}\right) \longrightarrow C_{\infty}\left(\mathbb{R}^{n}\right)$, the set of continuous functions vanishing at infinity. The operator $-p(x, D)$ is closable in $C_{\infty}\left(\mathbb{R}^{n}\right)$ and the closure is the generator of a Feller semigroup $\left(T_{t}\right)$, i.e. a strongly continuous sub-Markovian semigroup in $C_{\infty}\left(\mathbb{R}^{n}\right)$.

The conditions in Theorem 1.1 can be relaxed by a localization argument using the martingale problem as carried out in the last section. In this way we obtain

Theorem 1.2. Let $s(x, \xi)$ be as in Theorem $(1.1), m: \mathbb{R}^{n} \longrightarrow(0 ; 1]$ be $a C^{\infty}$-function and $p(x, \xi)$ as in (1.13).

Then $-p(x, D): C_{0}^{\infty}\left(\mathbb{R}^{n}\right) \longrightarrow C_{\infty}\left(\mathbb{R}^{n}\right)$ has an extension that generates a Feller semigroup $\left(T_{t}\right)$.

The proof of Theorem 1.1 relies on the theorem of Hille-Yosida. In particular for some $\tau \geq 0$ we have to find solutions of the equation

$$
(p(x, D)+\tau) u=f
$$

for sufficiently many right hand sides. We split the proof into a part concerning the existence of (weak) solutions and a part dealing with regularity. We treat both parts using typical techniques for pseudo differential operators, but in a suitably modified way. For that purpose we introduce an appropriate scale of anisotropic Sobolev spaces, which are defined in terms of the function $\lambda(\xi)$

$$
H^{s, \lambda}\left(\mathbb{R}^{n}\right)=\left\{u \in \mathcal{S}^{\prime}\left(\mathbb{R}^{n}\right):\|u\|_{s, \lambda}<\infty\right\}, \quad s \in \mathbb{R}
$$

where

$$
\|u\|_{s, \lambda}=\left(\int_{\mathbb{R}^{n}} \lambda^{2 s}(\xi)|\hat{u}(\xi)|^{2} d \xi\right)^{1 / 2}
$$

Note that $H^{s, \lambda}\left(\mathbb{R}^{n}\right)$ coincides with the space $H^{s / 2, a^{2}}\left(\mathbb{R}^{n}\right)$ defined in [12], in particular $H^{0, \lambda}\left(\mathbb{R}^{n}\right)=L^{2}\left(\mathbb{R}^{n}\right)$ and $C_{0}^{\infty}\left(\mathbb{R}^{n}\right)$ is dense in $H^{s, \lambda}\left(\mathbb{R}^{n}\right)$ for all $s \in \mathbb{R}$.

To prove existence of solutions we first apply a modified version of Friedrichs symmetrization that yields a sharp Gårding inequality, that is a lower bound for the corresponding bilinear form in terms of the lower order norm $\|\cdot\|_{\mu, \lambda}$. Then we show that the bilinear form is 
continuous on the space obtained by closing the symmetric part of the form and finally show

Theorem 1.3. Let $p(x, \xi)$ be as in Theorem 1.1 and $\tau>0$ sufficiently large. Then for any $f \in H^{-\mu, \lambda}\left(\mathbb{R}^{n}\right)$ there is a unique $u \in H^{\mu, \lambda}\left(\mathbb{R}^{n}\right)$ such that

$$
(p(x, D)+\tau) u=f
$$

Next we show that the operator $p(x, D)$ admits a (left-) parametrix, i.e. there is a symbol $q$ such that

$$
q(x, D) \circ p(x, D)=\mathrm{id}+r(x, D)
$$

where $r(x, D)$ is an operator of negative order, hence has smoothing properties. We then easily obtain the following regularity result.

Theorem 1.4. Let $p(x, \xi)$ as in Theorem 1.1 and $u$ be a solution of (1.14) for some $f \in H^{k, \lambda}\left(\mathbb{R}^{n}\right), k \geq 0$. Then for all $\varepsilon>0$ we have

$$
u \in H^{k+2 \mu-\varepsilon, \lambda}\left(\mathbb{R}^{n}\right) .
$$

\section{Symbolic calculus.}

In this section we recall some results from [9] and [10] where a symbolic calculus for pseudo differential operators with negative definite symbols is developed. Let again $a^{2}: \mathbb{R}^{n} \longrightarrow \mathbb{R}$ be a continuous negative definite reference function. Then by the Lévy-Khinchin formula $a^{2}$ has the unique representation

$$
a^{2}(\xi)=c+q(\xi)+\int_{\mathbb{R}^{n} \backslash\{0\}}(1-\cos (y, \xi)) \nu(d y),
$$

where $c$ is a nonnegative constant, $q$ is a positive definite (possibly degenerate) quadratic form and the so-called Lévy measure $\nu$ is a symmetric Borel measure on $\mathbb{R}^{n} \backslash\{0\}$ satisfying

$$
\int_{\mathbb{R}^{n} \backslash\{0\}} \frac{|y|^{2}}{1+|y|^{2}} \nu(d y)<\infty .
$$


Important examples of continuous negative definite functions are $\xi \longmapsto$ $|\xi|^{\alpha}, 0<\alpha \leq 2$ which lead to the symmetric $\alpha$-stable process, but they also illustrate that in general continuous negative definite functions are not differentiable. However if we consider a continuous negative definite function with a Lévy measure that has bounded support, that is $\operatorname{supp} \nu \subset B_{R}(0)$ for some $R>0$, then we have, see [9, Proposition 2.1] or [10, Proposition 1],

$$
a^{2} \in C^{\infty}\left(\mathbb{R}^{n}\right)
$$

and

$$
\left|\partial_{\xi}^{\alpha} a^{2}(\xi)\right| \leq c_{\alpha} a^{(2-\varrho(|\alpha|))}(\xi), \quad \varrho(k)=k \wedge 2, \alpha \in \mathbb{N}_{0}^{n},
$$

where the constants $c_{\alpha}$ depend only on the (finite) absolute moments of the Lévy measure of order greater or equal to 2 and the maximal eigenvalue of the quadratic form $q$. Supposing that a continuous negative definite symbol

$$
p: \mathbb{R}^{n} \times \mathbb{R}^{n} \longrightarrow \mathbb{R}
$$

also has Lévy measures supported in $B_{R}(0)$ and is controlled by $a^{2}$ in the sense that

$$
p(x, \xi) \leq c\left(1+a^{2}(\xi)\right),
$$

then using the notation of (1.8) the estimate (2.2) yields

$$
\left|\partial_{\xi}^{\alpha} p(x, \xi)\right| \leq c_{\alpha} a^{(2-\varrho(|\alpha|))}(\xi) \leq c_{\alpha} \lambda(\xi)^{(2-\varrho(|\alpha|))}
$$

and the constants $c_{\alpha}$ are independent of $x$. Therefore the condition $p \in S_{\varrho}^{2, \lambda}$, that is

$$
\left|\partial_{\xi}^{\alpha} \partial_{x}^{\beta} p(x, \xi)\right| \leq c_{\alpha, \beta} \lambda(\xi)^{2-\varrho(|\alpha|)}, \quad x \in \mathbb{R}^{n}, \xi \in \mathbb{R}^{n}, \alpha, \beta \in \mathbb{N}_{0}^{n}
$$

is nothing but the assumption that we have the same behaviour for the $x$-derivatives of the symbol.

Moreover we will use the larger class $S_{0}^{m, \lambda}$ which we obtain from (1.10) by replacing $\varrho$ by 0

$$
\left|\partial_{\xi}^{\alpha} \partial_{x}^{\beta} p(x, \xi)\right| \leq c_{\alpha, \beta} \lambda(\xi)^{m}, \quad x \in \mathbb{R}^{n}, \xi \in \mathbb{R}^{n}, \alpha, \beta \in \mathbb{N}_{0}^{n} .
$$

Note that in particular $\lambda^{m}(\xi)$ is an intrinsic example of a symbol in $S_{\varrho}^{m, \lambda}$.

In [9] a symbolic calculus for these classes of pseudo differential operators was developed similar to the results of Kumano-go [23] for 
symbols with general weight functions $\lambda$. Since our weight function $\lambda$ doesn't satisfy the differentiability properties which are assumed for the basic weight functions of [23] it does not fit into that framework. Thus modified proofs were necessary, using explicitly properties of negative definite functions. We will need the following results: For $p \in S_{0}^{m, \lambda}$ the operator $p(x, D)$ is a well-defined object that satisfies ([9, Proposition 3.2])

$$
p(x, D): \mathcal{S}\left(\mathbb{R}^{n}\right) \longrightarrow \mathcal{S}\left(\mathbb{R}^{n}\right) \text { continuously } .
$$

Therefore it makes sense to talk about composition of operators and formally adjoint operators.

Proposition 2.1 ([9, Corollaries 3.5, 3.11], [10, Theorem 2.4]). Let $p_{1} \in S_{0}^{m_{1}, \lambda}, p_{2} \in S_{0}^{m_{2}, \lambda}$. Then $p_{1}(x, D) \circ p_{2}(x, D)$ is again a pseudo differential operator with symbol in $S_{0}^{m_{1}+m_{2}, \lambda}$.

If moreover $p_{1} \in S_{\varrho}^{m_{1}, \lambda}, p_{2} \in S_{\varrho}^{m_{2}, \lambda}$ we have

$$
p_{1}(x, D) \circ p_{2}(x, D)=\left(p_{1} \cdot p_{2}\right)(x, D)+r(x, D),
$$

where the product of the symbols satisfies $p_{1} \cdot p_{2} \in S_{\varrho}^{m_{1}+m_{2}, \lambda}$ and $r \in$ $S_{0}^{m_{1}+m_{2}-1, \lambda}$.

By $p^{*}(x, D)$ we denote the formally adjoint of $p(x, D)$, which means

$$
\left(p^{*}(x, D) u, v\right)=(u, p(x, D) v), \quad u, v \in \mathcal{S}\left(\mathbb{R}^{n}\right),
$$

where $(\cdot, \cdot)$ is the $L^{2}$-inner product.

Proposition 2.2 ([9, ,Corollaries 3.6, 3.11], [10, Theorem 2.4]). Let $p \in$ $S_{0}^{m, \lambda}$. Then the formally adjoint $p^{*}(x, D)$ is again a pseudo differential operator with symbol in $S_{0}^{m, \lambda}$.

If moreover $p \in S_{\varrho}^{m, \lambda}$ we have

$$
p^{*}(x, D)=\bar{p}(x, D)+r^{\prime}(x, D)
$$

where the complex conjugate $\bar{p}$ of the symbol satisfies $\bar{p} \in S_{\varrho}^{m, \lambda}$ and $r^{\prime} \in S_{0}^{m-1, \lambda}$.

Therefore up to lower order terms composition and taking the formally adjoint have easy interpretations on the level of symbols. 
By duality the formally adjoint allows for $p \in S_{0}^{m, \lambda}$ to extend the operator continuously to

$$
p(x, D): \mathcal{S}^{\prime}\left(\mathbb{R}^{n}\right) \longrightarrow \mathcal{S}^{\prime}\left(\mathbb{R}^{n}\right)
$$

and we now can find a natural meaning of the order of an operator. Recall the definition of the spaces $H^{s, \lambda}\left(\mathbb{R}^{n}\right)$ in $(1.15),(1.16)$.

Proposition 2.3 ([9, Theorem 3.7], [10, Theorem 3]). Let $p \in S_{0}^{m, \lambda}$. Then for every $s \in \mathbb{R}$ the operator

$$
p(x, D): H^{s+m, \lambda}\left(\mathbb{R}^{n}\right) \longrightarrow H^{s, \lambda}\left(\mathbb{R}^{n}\right)
$$

is continuous.

Finally we will need the Friedrichs symmetrization, which in a modified form is also available in this context (see [9, Theorems 4.1, 4.4]).

Theorem 2.4. Let $p \in S_{\varrho}^{m, \lambda}$ be a real-valued non-negative symbol. Then there is a symbol $p_{F} \in S_{0}^{m, \lambda}$ such that $p-p_{F} \in S_{0}^{m-1, \lambda}$ and the operator $p_{F}(x, D)$, the Friedrichs symmetrization, is a symmetric non-negative definite operator in $L^{2}\left(\mathbb{R}^{n}\right)$, i.e.

$$
\left(p_{F}(x, D) u, u\right) \geq 0
$$

for all $u \in \mathcal{S}\left(\mathbb{R}^{n}\right)$.

\section{Existence of solutions.}

In this section let $s \in S_{\varrho}^{2, \lambda}$ be a negative definite elliptic symbol, i.e. there is a $\delta>0$ such that

$$
s(x, \xi) \geq \delta \lambda^{2}(\xi), \quad x, \xi \in \mathbb{R}^{n},
$$

and let $m: \mathbb{R}^{n} \longrightarrow(0 ; 1], m \in C_{b}^{\infty}\left(\mathbb{R}^{n}\right)$ be as in Theorem 1.1, that is for

$$
M=\sup _{x \in \mathbb{R}^{n}} m(x), \quad \mu=\inf _{x \in \mathbb{R}^{n}} m(x),
$$


we assume

$$
M-\mu<\frac{1}{2}, \quad \mu>0
$$

and consider

$$
p(x, \xi)=s(x, \xi)^{m(x)} .
$$

The first property we have to check is whether $p(x, \xi)$ is a symbol in the symbol classes $S_{\varrho}^{m, \lambda}$. Since the exponent $m(x)$ depends on $x$, differentiation of $p$ with respect to $x$ yields certain logarithmic term of $s(x, \xi)$. In the case of symbols (1.5) this can be treated by considering the Hörmander classes $S_{\varrho, \delta}^{m}$ with $\delta>0$. A similar procedure for symbols in $S_{\varrho}^{m, \lambda}$ causes problems for the symbolic calculus, since the order of the derivatives $\partial_{\xi}^{\alpha} p(x, \xi)$ does not decrease arbitrarily as $|\alpha| \rightarrow \infty$. Therefore it is more convenient to capture the effect of the $x$-derivatives by slightly increasing the order of the symbol, i.e. $p \in S_{\varrho}^{2 M+\varepsilon, \lambda}$ for $\varepsilon>0$. First we need

Lemma 3.1. Let $G, K, L: \mathbb{R}^{N} \longrightarrow \mathbb{R}$ be $C^{\infty}$-functions, $G>0, L \neq 0$. Then we have for $\gamma \in \mathbb{N}_{0}^{N}, l=|\gamma|$

i)

$$
\partial^{\gamma} \exp K=\exp K \sum_{\substack{\gamma_{1}+\cdots+\gamma_{l^{\prime}}=\gamma \\ l^{\prime}=0,1, \ldots, l}} c_{\left\{\gamma_{i}\right\}} \prod_{i=1}^{l^{\prime}} \partial^{\gamma_{i}} K
$$

ii)

$$
\partial^{\gamma} \log G=\sum_{\gamma_{1}+\cdots+\gamma_{l}=\gamma} c_{\left\{\gamma_{i}\right\}}^{\prime} \prod_{i=1}^{l} \frac{\partial^{\gamma_{i}} G}{G}, \quad \text { if } \gamma \neq 0
$$

iii)

$$
\partial^{\gamma} \frac{1}{L}=\frac{1}{L} \sum_{\gamma_{1}+\cdots+\gamma_{l}=\gamma} c_{\left\{\gamma_{i}\right\}}^{\prime \prime} \prod_{i=1}^{l} \frac{\partial^{\gamma_{i}} L}{L} .
$$

The summation is taken over all choices of multiindices $\gamma_{1}, \ldots, \gamma_{l^{\prime}} \in$ $\mathbb{N}_{0}^{N}$ and $\gamma_{1}, \ldots, \gamma_{l} \in \mathbb{N}_{0}^{N}$, respectively, that have sum $\gamma$. The constants $c_{\left\{\gamma_{i}\right\}}, c_{\left\{\gamma_{i}\right\}}^{\prime}$ and $c_{\left\{\gamma_{i}\right\}}^{\prime \prime}$ depend on the choice of the multiindices. 
The proof by induction is an elementary application of the chain rule. See also Fraenkel [6] for general higher order chain rules in higher dimensions.

We now are able to prove

Proposition 3.2. Let $p(x, \xi)$ be as above. Then for all $\varepsilon>0$

$$
\left|\partial_{\xi}^{\alpha} \partial_{x}^{\beta} p(x, \xi)\right| \leq c_{\alpha \beta \varepsilon} p(x, \xi) \lambda^{-\varrho(|\alpha|)+\varepsilon}(\xi) .
$$

In particular $p \in S_{\varrho}^{2 M+\varepsilon, \lambda}$.

ProOF. We have to estimate the derivatives

$$
\partial_{\xi}^{\alpha} \partial_{x}^{\beta} p(x, \xi)=\partial_{\xi}^{\alpha} \partial_{x}^{\beta} s(x, \xi)^{m(x)}=\partial_{\xi}^{\alpha} \partial_{x}^{\beta} \exp (m(x) \log s(x, \xi)) .
$$

We apply Lemma 3.1.i) with $N=2 n, \gamma=(\alpha, \beta), l=|\alpha|+|\beta|$. Thus

$$
\begin{gathered}
\left|\partial_{\xi}^{\alpha} \partial_{x}^{\beta} p(x, \xi)\right| \leq \exp (m(x) \log s(x, \xi)) \\
\cdot\left|\sum_{\substack{\alpha_{1}+\cdots+\alpha_{l^{\prime}}=\alpha \\
\beta_{1}+\cdots+\beta_{l^{\prime}}=\beta \\
l^{\prime}=0,1, \ldots, l}} c_{\left\{\alpha_{i}, \beta_{i}\right\}} \prod_{i=1}^{l^{\prime}} t_{\alpha_{i} \beta_{i}}(x, \xi)\right|,
\end{gathered}
$$

where

$$
\begin{aligned}
t_{\alpha_{i} \beta_{i}}(x, \xi) & =\partial_{\xi}^{\alpha_{i}} \partial_{x}^{\beta_{i}}(m(x) \log s(x, \xi)) \\
& =\sum_{\beta_{i}^{\prime} \leq \beta_{i}}\left(\begin{array}{c}
\beta_{i} \\
\beta_{i}^{\prime}
\end{array}\right) \partial_{x}^{\beta_{i}-\beta_{i}^{\prime}} m(x) \partial_{\xi}^{\alpha_{i}} \partial_{x}^{\beta_{i}^{\prime}} \log s(x, \xi) .
\end{aligned}
$$

Again by Lemma 3.1.ii), if $k=\left|\alpha_{i}\right|+\left|\beta_{i}^{\prime}\right| \neq 0$

$$
\partial_{\xi}^{\alpha_{i}} \partial_{x}^{\beta_{i}^{\prime}} \log s(x, \xi)=\sum_{\substack{\widetilde{\alpha}_{1}+\cdots+\widetilde{\alpha}_{k}=\alpha_{i} \\ \widetilde{\beta}_{1}+\cdots+\widetilde{\beta}_{k}=\beta_{i}^{\prime}}} c_{\left\{\widetilde{\alpha}_{j}, \widetilde{\beta}_{j}\right\}} \prod_{j=1}^{k} \frac{\partial_{\xi}^{\widetilde{\alpha}_{j}} \partial_{x}^{\widetilde{\beta}_{j}} s(x, \xi)}{s(x, \xi)} .
$$

Since $s(x, \xi)$ is an elliptic symbol in $S_{\varrho}^{2, \lambda}$ we find

$$
\begin{aligned}
\left|\partial_{\xi}^{\alpha_{i}} \partial_{x}^{\beta_{i}^{\prime}} \log s(x, \xi)\right| & \leq c_{\alpha_{i} \beta_{i}^{\prime}} \sum_{\substack{\widetilde{\alpha}_{1}+\cdots+\widetilde{\alpha}_{k}=\alpha_{i} \\
\widetilde{\beta}_{1}+\cdots+\widetilde{\beta}_{k}=\beta_{i}^{\prime}}} \prod_{j=1}^{k} \lambda^{-\varrho\left(\left|\widetilde{\alpha}_{j}\right|\right)}(\xi) \\
& \leq c_{\alpha_{i} \beta_{i}^{\prime}} \lambda^{-\varrho\left(\left|\alpha_{i}\right|\right)}(\xi)
\end{aligned}
$$


where we used the subadditivity of $\varrho$ in the last step. Moreover we always have $|\log s(x, \xi)| \leq c \log \lambda(\xi) \leq c_{\varepsilon} \lambda^{\varepsilon / l}(\xi)$. Since $m \in C_{b}^{\infty}\left(\mathbb{R}^{n}\right)$ we therefore get from (3.6)

$$
\left|t_{\alpha_{i} \beta_{i}}(x, \xi)\right| \leq c_{\alpha_{i} \beta_{i} \varepsilon} \begin{cases}\lambda^{-\varrho\left(\left|\alpha_{i}\right|\right)}(\xi), & \alpha_{i} \neq 0 \\ \lambda^{\varepsilon / l}(\xi), & \alpha_{i}=0\end{cases}
$$

and finally by (3.5)

$$
\begin{aligned}
& \left|\partial_{\xi}^{\alpha} \partial_{x}^{\beta} p(x, \xi)\right| \\
& \leq p(x, \xi) c_{\alpha \beta \varepsilon} \sum_{\substack{\alpha_{1}+\cdots+\alpha_{l^{\prime}}=\alpha \\
\beta_{1}+\cdots+\beta_{l^{\prime}}=\beta \\
l^{\prime}=0,1, \ldots, l}}\left(\prod_{\substack{i=1, \ldots, l^{\prime} \\
\alpha_{i} \neq 0}} \lambda^{-\varrho\left(\left|\alpha_{i}\right|\right)}(\xi) \prod_{\substack{i=1, \ldots, l^{\prime} \\
\alpha_{i}=0}} \lambda^{\varepsilon / l}(\xi)\right) \\
& \quad \leq p(x, \xi) c_{\alpha \beta \varepsilon} \lambda^{-\varrho(|\alpha|)+\varepsilon}(\xi)
\end{aligned}
$$

The second statement follows immediately from $p(x, \xi) \leq c \lambda^{2 M}(\xi)$.

We want to consider the equation

$$
(p(x, D)+\tau) u=f,
$$

for $\tau \geq 0$. Let $p_{\tau}(x, \xi)=p(x, \xi)+\tau$ and

$$
B_{\tau}(u, v)=\left(p_{\tau}(x, D) u, v\right), \quad u, v \in C_{0}^{\infty}\left(\mathbb{R}^{n}\right),
$$

be the associated bilinear form. Here $(\cdot, \cdot)$ is the inner product in $L^{2}\left(\mathbb{R}^{n}\right)$. We note

Lemma 3.3. Let $q \in S_{0}^{2 m, \lambda}$. Then the bilinear form

$$
(u, v) \longmapsto(q(x, D) u, v), \quad u, v \in C_{0}^{\infty}\left(\mathbb{R}^{n}\right),
$$

has a continuous extension to $H^{m, \lambda}\left(\mathbb{R}^{n}\right)$.

This follows immediately from

$$
|(q(x, D) u, v)|=\left|\left(\lambda^{-m}(D) \circ q(x, D) u, \lambda^{m}(D) v\right)\right| \leq c\|u\|_{m, \lambda}\|v\|_{m, \lambda}
$$

by Cauchy-Schwarz inequality, since by Proposition 2.1 both $\lambda^{-m}(D) \circ$ $q(x, D)$ and $\lambda^{m}(D)$ are operators of order $m$. 
Observe that by the ellipitcity of $s$ there is a $\delta^{\prime}>0$ such that

$$
p(x, \xi) \geq \delta^{\prime} \lambda^{2 \mu}(\xi) .
$$

Theorem 3.4. Let $\varepsilon>0$ such that $M-\mu+\varepsilon<1 / 2$ and let $M^{\prime}=$ $M+\varepsilon / 2$. Then $B_{\tau}$ extends continuously to $H^{M^{\prime}, \lambda}\left(\mathbb{R}^{n}\right)$ and if $\tau$ is sufficiently large, the lower estimate

$$
B_{\tau}(u, u) \geq \frac{\delta^{\prime}}{2}\|u\|_{\mu, \lambda}^{2}
$$

holds.

Proof. The first statement is immediate from Lemma 3.3 since $p_{\tau} \in$ $S_{\varrho}^{2 M^{\prime}, \lambda}$. Let $Q(x, \xi)=p(x, \xi)-\delta^{\prime} \lambda^{2 \mu}(\xi) \in S_{\varrho}^{2 M^{\prime}, \lambda}$. By (3.8) we have $Q(x, \xi) \geq 0$ and hence by Theorem 2.4 we know that the Friedrichs symmetrization $Q_{F}(x, D)$ is a symmetric nonnegative operator with symbol $Q_{F} \in S_{0}^{2 M^{\prime}, \lambda}$ such that $r=Q-Q_{F} \in S_{0}^{2 M^{\prime}-1, \lambda}$.

Then by Lemma 3.3

$$
\begin{aligned}
(p(x, D) u, u)-\delta^{\prime}\|u\|_{\mu, \lambda}^{2} & =(Q(x, D) u, u) \\
& =\left(Q_{F}(x, D) u, u\right)+(r(x, D) u, u) \\
& \geq-c\|u\|_{M^{\prime}-1 / 2, \lambda}^{2} \\
& \geq-\frac{\delta^{\prime}}{2}\|u\|_{\mu, \lambda}^{2}-c\left(\delta^{\prime}\right)\|u\|_{0}^{2} .
\end{aligned}
$$

Note that the first inequality represents a modified form of the sharp Gårding inequality. In the last step we used the fact that $M^{\prime}-1 / 2<\mu$ and the inequality

$$
\|u\|_{s_{2}, \lambda} \leq A\|u\|_{s_{1}, \lambda}+c(A)\|u\|_{s_{3}, \lambda}
$$

for $s_{1} \geq s_{2} \geq s_{3}$ and any $A>0$ (see [8, (2.9)]). Choosing $\tau \geq c\left(\delta^{\prime}\right)$ proves (3.9).

$B_{\tau}$ is a continuous bilinear form on $H^{M^{\prime}, \lambda}\left(\mathbb{R}^{n}\right)$ but satisfies a lower bound only with respect to a lower order norm, which of course reflects the character of varying order. To get a weak solution of (3.7) in terms of this form $B_{\tau}$ we will use a method which is known in the case of 
degenerate elliptic differential operators, see Louhivaara, Simader [20], [21]. For that purpose let

$$
\widetilde{B}_{\tau}(u, v)=\frac{1}{2}\left(B_{\tau}(u, v)+B_{\tau}(v, u)\right), \quad u, v \in H^{M^{\prime}, \lambda}\left(\mathbb{R}^{n}\right),
$$

be the symmetric part of $B_{\tau}$. Then obviously

$$
\left|\widetilde{B}_{\tau}(u, v)\right| \leq c\|u\|_{M^{\prime}, \lambda}\|v\|_{M^{\prime}, \lambda}
$$

and

$$
\widetilde{B}_{\tau}(u, u) \geq \frac{\delta^{\prime}}{2}\|u\|_{\mu, \lambda}^{2}
$$

Therefore $\widetilde{B}_{\tau}$ is a symmetric bilinear form on $H^{M^{\prime}, \lambda}\left(\mathbb{R}^{n}\right)$ which by (3.11) is positive and not degenerate, i.e. $\widetilde{B}_{\tau}(u, u)=0$ if and only if $u=0$, that is $\widetilde{B}_{\tau}$ is an inner product. Of course in general $H^{M^{\prime}, \lambda}\left(\mathbb{R}^{n}\right)$ is not complete with respect to this inner product. By $H^{p_{\tau}}$ we denote the completion of $H^{M^{\prime}, \lambda}\left(\mathbb{R}^{n}\right)$ with respect to the norm $\|\cdot\|_{p_{\tau}}=\widetilde{B}_{\tau}^{1 / 2}$. Then $\left(H^{p_{\tau}},\|\cdot\|_{p_{\tau}}\right)$ is a Hilbert space. By (3.10) and (3.11) we can construct the completion in such a way that the continuous and dense embeddings

$$
H^{M^{\prime}, \lambda}\left(\mathbb{R}^{n}\right) \hookrightarrow H^{p_{\tau}} \hookrightarrow H^{\mu, \lambda}\left(\mathbb{R}^{n}\right)
$$

hold.

Lemma 3.5. $B_{\tau}$ is a continuous bilinear form on $\left(H^{p_{\tau}},\|\cdot\|_{p_{\tau}}\right)$.

Proof. Since $p_{\tau}(x, \xi)$ is real-valued, Proposition 2 yields

$$
\begin{aligned}
\frac{1}{2}\left(p_{\tau}(x, D)+p_{\tau}^{*}(x, D)\right) & =\frac{1}{2}\left(p_{\tau}(x, D)+\bar{p}_{\tau}(x, D)\right)+r_{1}(x, D) \\
& =p_{\tau}(x, D)+r_{1}(x, D)
\end{aligned}
$$

where $r_{1} \in S_{0}^{2 M^{\prime}-1, \lambda}$ and therefore for $u, v \in C_{0}^{\infty}\left(\mathbb{R}^{n}\right)$

$$
\begin{aligned}
\left|B_{\tau}(u, v)\right| & =\left|\left(p_{\tau}(x, D) u, v\right)\right| \\
& \leq\left|\frac{1}{2}\left(\left(p_{\tau}(x, D)+p_{\tau}^{*}(x, D)\right) u, v\right)\right|+\left|\left(r_{1}(x, D) u, v\right)\right| \\
& =\left|\widetilde{B}_{\tau}(u, v)\right|+\left|\left(r_{1}(x, D) u, v\right)\right| .
\end{aligned}
$$


$\widetilde{B}_{\tau}$ is continuous on $H^{p_{\tau}}$ by definition and by Lemma $3.3\left(r_{1}(x, D) u, v\right)$ is continuous on $H^{M^{\prime}-1 / 2, \lambda}\left(\mathbb{R}^{n}\right)$ and therefore also on $H^{p_{\tau}}$, because by $M^{\prime}-1 / 2<\mu$ we have the continuous embedding

$$
H^{p_{\tau}} \hookrightarrow H^{\mu, \lambda}\left(\mathbb{R}^{n}\right) \hookrightarrow H^{M^{\prime}-1 / 2, \lambda}\left(\mathbb{R}^{n}\right)
$$

REMARK. In other words $B_{\tau}$ with domain $H^{p_{\tau}}$ is a sectorial form in the sense of Kato [16, VI.2].

It is now easy to give a

Proof of Theorem 1.3. By Lemma 3.5 we know that $B_{\tau}$ is a continuous and by definition coercive bilinear form on $H^{p_{\tau}}$. Thus by the theorem of Lax-Milgram for any $f$ in the dual space $\left(H^{p_{\tau}}\right)^{\prime}$ there is a unique $u \in H^{p_{\tau}}$ such that

$$
B_{\tau}(u, v)=\langle f, v\rangle, \quad \text { for all } v \in H^{p_{\tau}} .
$$

We choose a sequence $\left\{u_{k}\right\}$ in $C_{0}^{\infty}\left(\mathbb{R}^{n}\right)$ which converges to $u$ in $H^{p_{\tau}}$ and consequently also in $H^{\mu, \lambda}\left(\mathbb{R}^{n}\right)$. Note that for any $v \in C_{0}^{\infty}\left(R^{n}\right)$ the map $u \longmapsto(u, v)$ has a continuous extension to $H^{\mu-2 M^{\prime}, \lambda}\left(\mathbb{R}^{n}\right)$ and $p_{\tau}(x, D)$ : $H^{\mu, \lambda}\left(\mathbb{R}^{n}\right) \longrightarrow H^{\mu-2 M^{\prime}, \lambda}\left(\mathbb{R}^{n}\right)$ is continuous. Thus the equation

$$
\left(p_{\tau}(x, D) u_{k}, v\right)=B_{\tau}\left(u_{k}, v\right), \quad v \in C_{0}^{\infty}\left(\mathbb{R}^{n}\right),
$$

yields for $k \longrightarrow \infty$

$$
\left\langle p_{\tau}(x, D) u, v\right\rangle=B_{\tau}(u, v)=\langle f, v\rangle, \quad \text { for all } v \in C_{0}^{\infty}\left(\mathbb{R}^{n}\right),
$$

and therefore

$$
p_{\tau}(x, D) u=f .
$$

In particular because of the embeddings

$$
H^{p_{\tau}} \hookrightarrow H^{\mu, \lambda}\left(\mathbb{R}^{n}\right) \quad \text { and } \quad H^{-\mu, \lambda}\left(\mathbb{R}^{n}\right) \hookrightarrow\left(H^{p_{\tau}}\right)^{\prime}
$$

we have a unique weak solution $u \in H^{\mu, \lambda}\left(\mathbb{R}^{n}\right)$ of equation (3.7) for any $f \in H^{-\mu, \lambda}\left(\mathbb{R}^{n}\right)$. 


\section{Regularity of solutions.}

Let $p(x, \xi)$ as in Section 3 and $\varepsilon>0$ such that $M-\mu+\varepsilon<1 / 2$. Our aim is to construct a (left-) parametix to the operator $p_{\tau}(x, D)$, that is an inverse modulo a smoothing operator. From the existence of such parametrix we then easily obtain regularity for the solution of equation (3.7).

The symbolic calculus for $S_{\varrho}^{m, \lambda}$ does not yield expansion series with remainder terms of arbitrarily small order. But it turns out to be sufficient to use a first order expansion to get a smoothing remainder term, i.e. an operator which is order improving with respect to the scale of Sobolev spaces $H^{s, \lambda}\left(\mathbb{R}^{n}\right)$.

Define

$$
q_{\tau}(x, \xi)=\frac{1}{p_{\tau}(x, \xi)} .
$$

Lemma 4.1. We have

$$
q_{\tau} \in S_{\varrho}^{-2 \mu+\varepsilon, \lambda}
$$

Proof. We apply Lemma 3.1.iii) to estimate the derivatives of $q_{\tau}(x, \xi)$ and it follows with $l=|\alpha|+|\beta|$

$$
\left|\partial_{\xi}^{\alpha} \partial_{x}^{\beta} q_{\tau}(x, \xi)\right| \leq \frac{1}{p_{\tau}(x, \xi)} \sum_{\substack{\alpha_{1}+\cdots+\alpha_{l}=\alpha \\ \beta_{1}+\cdots+\beta_{l}=\beta}} c_{\left\{\alpha_{i}, \beta_{i}\right\}} \prod_{i=1}^{l}\left|\frac{\partial_{\xi}^{\alpha_{i}} \partial_{x}^{\beta_{i}} p_{\tau}(x, \xi)}{p_{\tau}(x, \xi)}\right|
$$

By (3.4) we have

$$
\left|\frac{\partial_{\xi}^{\alpha_{i}} \partial_{x}^{\beta_{i}} p_{\tau}(x, \xi)}{p_{\tau}(x, \xi)}\right| \leq c_{\alpha_{i} \beta_{i} \varepsilon} \lambda^{-\varrho\left(\left|\alpha_{i}\right|\right)+\varepsilon}(\xi)
$$

for any $\varepsilon>0$ and therefore by (3.1)

$$
\left|\partial_{\xi}^{\alpha} \partial_{x}^{\beta} q_{\tau}(x, \xi)\right| \leq c_{\alpha \beta \varepsilon} \lambda^{-2 \mu}(\xi) \lambda^{-\varrho(|\alpha|)+\varepsilon}(\xi),
$$

for all $\varepsilon>0$ by the subadditivity of $\varrho$.

Now the proof of Theorem 1.4 is almost immediate. 
Proof of TheOREM 1.4. Let $f \in H^{k, \lambda}\left(\mathbb{R}^{n}\right)$ and $u$ be the solution of (3.7) which is in $H^{\mu, \lambda}\left(\mathbb{R}^{n}\right)$ by Theorem 1.3. Then Proposition 2.1 gives

$$
q_{\tau}(x, D) \circ p_{\tau}(x, D)=\mathrm{id}+r(x, D),
$$

where $r \in S_{0}^{-t, \lambda}$ for

$$
-t=(-2 \mu+\varepsilon)+(2 M+\varepsilon)-1=2\left(M-\mu+\varepsilon-\frac{1}{2}\right)<0 .
$$

We apply (4.1) to $u$ and obtain

$$
u=q_{\tau}(x, D) \circ p_{\tau}(x, D) u-r(x, D) u=q_{\tau}(x, D) f-r(x, D) u .
$$

We have $q_{\tau}(x, D) f \in H^{k+2 \mu-\varepsilon, \lambda}\left(\mathbb{R}^{n}\right)$ and $r(x, D)$ is order improving, that is $u \in H^{\mu, \lambda}\left(\mathbb{R}^{n}\right)$ implies $r(x, D) u \in H^{\mu+t, \lambda}\left(\mathbb{R}^{n}\right)$ and hence $u \in H^{(\mu+t) \wedge(k+2 \mu-\varepsilon), \lambda}\left(\mathbb{R}^{n}\right)$. Applying this argument recursively finally gives $u \in H^{k+2 \mu-\varepsilon, \lambda}\left(\mathbb{R}^{n}\right)$.

In order to find solutions of (3.7) also in $C_{\infty}\left(\mathbb{R}^{n}\right)$ we need a Sobolev embedding for $H^{s, \lambda}\left(\mathbb{R}^{n}\right)$ in $C_{\infty}\left(\mathbb{R}^{n}\right)$. Recall that for the reference function $a^{2}$ we have imposed the assumption (1.7)

$$
a^{2}(\xi) \geq c|\xi|^{r}, \quad|\xi| \text { large }
$$

which was not used up to now. In fact it is easy to see that under (1.7) $H^{s, \lambda}\left(\mathbb{R}^{n}\right)$ is continuously embedded into the ordinary Sobolev space $H^{r s / 2}\left(\mathbb{R}^{n}\right)$ and it follows (see [8, Section 2])

$$
H^{s, \lambda}\left(\mathbb{R}^{n}\right) \hookrightarrow C_{\infty}\left(\mathbb{R}^{n}\right), \quad \text { if } s>\frac{n}{r} .
$$

Let us give the

Proof of Theorem 1.1. Let again $\varepsilon>0$ satisfy $M-\mu+\varepsilon<1 / 2$. We know that $p \in S_{\varrho}^{2 M+\varepsilon, \lambda}$. Choose $k>0$ such that $k>n / r$. Then $H^{k+2 M+\varepsilon, \lambda}\left(\mathbb{R}^{n}\right)$ and $H^{k, \lambda}\left(\mathbb{R}^{n}\right)$ can be considered as dense subspaces of $C_{\infty}\left(\mathbb{R}^{n}\right)$ and it follows

i) that

$$
-p(x, D): H^{k+2 M+\varepsilon, \lambda}\left(\mathbb{R}^{n}\right) \longrightarrow H^{k, \lambda}\left(\mathbb{R}^{n}\right)
$$


is a densely defined operator in $C_{\infty}\left(\mathbb{R}^{n}\right)$.

Moreover $p$ is a continuous negative definite symbol. Hence by the result of Courrège, [4, Théorème 3.5$]$, we know that $-p(x, D)$ satisfies the positive maximum principle as an operator with domain $C_{0}^{\infty}\left(\mathbb{R}^{n}\right)$. By an approximation argument, see Jacob [13, Theorem 9.3], we see that

ii) $-p(x, D)$ satisfies the positive maximum principle also on $H^{k+2 M+\varepsilon, \lambda}\left(\mathbb{R}^{n}\right)$.

Finally let $\tau>0$ be sufficiently large and $f \in H^{k+2(M-\mu+\varepsilon), \lambda}\left(\mathbb{R}^{n}\right)$. Then by Theorem 1.3 and Theorem 1.4 we know that there is a $u \in$ $H^{k+2(M-\mu+\varepsilon)+2 \mu-\varepsilon, \lambda}\left(\mathbb{R}^{n}\right)=H^{k+2 M+\varepsilon, \lambda}\left(\mathbb{R}^{n}\right)$ such that

$$
p_{\tau}(x, D) u=f .
$$

In other words

iii) The range of the operator $p_{\tau}(x, D)=\tau-(-p(x, D))$ with domain $H^{k+2 M+\varepsilon, \lambda}\left(\mathbb{R}^{n}\right)$ contains $H^{k+2(M-\mu+\varepsilon), \lambda}\left(\mathbb{R}^{n}\right)$ and is therefore dense in $C_{\infty}\left(\mathbb{R}^{n}\right)$.

By the theorem of Hille-Yosida, see [5, Theorem 4.2.2], the conditions i)-iii) imply that the closure of $\left(-p(x, D), H^{k+2 M+\varepsilon, \lambda}\left(\mathbb{R}^{n}\right)\right)$ generates a Feller semigroup $\left(T_{t}\right)$.

But $C_{0}^{\infty}\left(\mathbb{R}^{n}\right)$ is dense in $H^{k+2 M+\varepsilon, \lambda}\left(\mathbb{R}^{n}\right), \quad-p(x, D)$ maps $H^{k+2 M+\varepsilon, \lambda}\left(\mathbb{R}^{n}\right)$ continuously into $H^{k, \lambda}\left(\mathbb{R}^{n}\right)$ and both $H^{k+2 M+\varepsilon, \lambda}\left(\mathbb{R}^{n}\right)$ and $H^{k, \lambda}\left(\mathbb{R}^{n}\right)$ are continuously embedded in $C_{\infty}\left(\mathbb{R}^{n}\right)$. Therefore this closure coincides with the closure of $\left(-p(x, D), C_{0}^{\infty}\left(\mathbb{R}^{n}\right)\right)$.

REMARK. By Lemma 3.5 we know that the bilinear form $B_{\tau}$ with domain $H^{p_{\tau}}$ is a closed coercive form in $L^{2}\left(\mathbb{R}^{n}\right)$ in the sense of Ma, Röckner [24]. We deduce that therefore $\left(T_{t}\right)$ is also a strongly continuous semigroup on $L^{2}\left(\mathbb{R}^{n}\right)$. The sub-Markovian property of $\left(T_{t}\right)$ hence implies that $B_{\tau}$ has the contraction property in [24, I.4.4], and is a semi-Dirichlet form.

\section{Localization by the martingale problem.}

The restriction (1.12) for the oscillation of the exponent function $m(x)$ implies in particular that the bilinear form $B_{\tau}$ is continuous with respect to its symmetric part, i.e. sectorial and therefore is necessary 
in the above approach. We can avoid this condition as well as the boundedness of the derivatives of $m(x)$ if we use an approach via the martingale problem. This is mainly due to the fact that well-posedness of the martingale problem is closely related to the property that the operator generates a Feller semigroup, see [30].

By a solution of the martingale problem for the operator $-p(x, D)$ we denote a probability measure $P \in \mathcal{M}_{1}\left(D_{\mathbb{R}^{n}}\right)$ on the path space $D_{\mathbb{R}^{n}}$ of all càdlàg-functions $\omega:[0 ; \infty) \longrightarrow \mathbb{R}^{n}$ such that for all $\varphi \in C_{0}^{\infty}\left(\mathbb{R}^{n}\right)$

$$
\varphi\left(X_{t}\right)-\int_{0}^{t}(-p(x, D) \varphi)\left(X_{s}\right) d s, \quad t \geq 0,
$$

is an $\left\{\mathcal{F}_{t}\right\}$-martingale, where $X_{t}(\omega)=\omega(t)$ and $\mathcal{F}_{t}=\sigma\left(X_{s}: s \leq t\right)$. The martingale problem is called well-posed if for every given initial distribution $P_{X_{0}} \in \mathcal{M}_{1}\left(\mathbb{R}^{n}\right)$ there is a unique solution.

In particular the following result of van Casteren [30, Proposition 2.6], holds true.

Theorem 5.1. Let $-p(x, D)$ be a densely defined linear operator in $C_{\infty}\left(\mathbb{R}^{n}\right)$ for which the martingale problem is well-posed. Then $-p(x, D)$ has a unique extension that generates a Feller semigroup.

REMARK. Let us first note that we may restrict to the conservative case, that is we may consider the symbol $\widetilde{p}(x, \xi):=p(x, \xi)-p(x, 0)$. Both $p(x, \xi)$ and $\widetilde{p}(x, \xi)$ are negative definite symbols and $x \mapsto p(x, 0)$ is a bounded continuous function. Therefore both $-p(x, D)$ and $-\widetilde{p}(x, D)$ satisfy the positive maximum principle and their difference is a bounded operator in $C_{\infty}\left(\mathbb{R}^{n}\right)$. By a standard perturbation result for generators of (Feller-) semigroups hence $-\widetilde{p}(x, D)$ generates a Feller semigroup if and only if $-p(x, D)$ does.

The key result we need in this section is ([7, Theorem 7.1], see also [5, Chapter 4.6]).

Theorem 5.2. Let $p, p_{k}: \mathbb{R}^{n} \times \mathbb{R}^{n} \longrightarrow \mathbb{R}, k \in \mathbb{N}$, be continuous negative definite symbols such that $p(x, D), p_{k}(x, D): C_{0}^{\infty}\left(\mathbb{R}^{n}\right) \longrightarrow$ $C_{b}\left(\mathbb{R}^{n}\right)$ and let $\left\{U_{k}\right\}_{k \in \mathbb{N}}$ be an open covering of $\mathbb{R}^{n}$ such that

$$
p(x, \xi)=p_{k}(x, \xi), \quad \text { for all } x \in U_{k}, \xi \in \mathbb{R}^{n} .
$$

If the martingale problem for $-p(x, D)$ has a solution for all initial distributions and the martingale problem for $-p_{k}(x, D)$ is well-posed 
for all $k \in \mathbb{N}$, then the martingale problem for $-p(x, D)$ is well-posed, too.

Proof of TheOrem 1.2. Let $p(x, \xi)$ be as in Theorem 1.2, i.e. $p(x, \xi)=s(x, \xi)^{m(x)}$, where $s \in S_{\varrho}^{2, \lambda}$ is a negative definite symbol which is elliptic in the sense of (1.11) and $m$ is a $C^{\infty}$-function on $\mathbb{R}^{n}$ with values in $(0 ; 1]$. Then

$$
\widetilde{p}(x, \xi):=p(x, \xi)-p(x, 0)
$$

is a negative definite symbol such that $\widetilde{p}(x, 0)=0$ and for a suitable $c \geq 0$

$$
\widetilde{p}(x, \xi) \leq c\left(1+|\xi|^{2}\right) .
$$

Thus by [8, Theorem 3.2], there is a solution to the martingale problem for $-\widetilde{p}(x, D)$ for any initial condition.

Next fix $x_{0} \in \mathbb{R}^{n}$ and choose open relatively compact neighbourhoods $U_{x_{0}}, V_{x_{0}}$ of $x_{0}$ such that $x_{0} \in U_{x_{0}} \subset \overline{U_{x_{0}}} \subset V_{x_{0}}$ and

$$
\left|m(x)-m\left(x_{0}\right)\right|<\frac{1}{5}, \quad \text { for all } x \in V_{x_{0}} .
$$

Let $\varphi_{x_{0}} \in C_{0}^{\infty}\left(\mathbb{R}^{n}\right)$ such that $0 \leq \varphi_{x_{0}} \leq 1, \varphi_{x_{0}}=1$ in $U_{x_{0}}$ and $\operatorname{supp} \varphi_{x_{0}} \subset V_{x_{0}}$ and define

$$
m_{x_{0}}(x)=\varphi_{x_{0}}(x) m(x)+\left(1-\varphi_{x_{0}}(x)\right) m\left(x_{0}\right) .
$$

Then

$$
\begin{gathered}
m_{x_{0}} \in C_{b}^{\infty}\left(\mathbb{R}^{n}\right), \\
\inf _{x \in \mathbb{R}^{n}} m_{x_{0}}(x)>0, \\
\sup _{x \in \mathbb{R}^{n}} m_{x_{0}}(x)-\inf _{x \in \mathbb{R}^{n}} m_{x_{0}}(x)<\frac{1}{2},
\end{gathered}
$$

and therefore the symbol

$$
p_{x_{0}}(x, \xi)=s(x, \xi)^{m_{x_{0}}(x)}
$$

satisfies the conditions of Theorem 1.1 and $-p_{x_{0}}(x, D)$ has an extension that generates a Feller semigroup. By the above remark the same holds true for $-\widetilde{p}_{x_{0}}(x, D)$, where

$$
\widetilde{p}_{x_{0}}(x, \xi)=\widetilde{p}_{x_{0}}(x, \xi)-\widetilde{p}_{x_{0}}(x, 0) .
$$


It is well-known that for a given initial distribution generators of Feller semigroups have at most one solution to the martingale problem (see for example [5, Corollary 4.4.4]). Thus again by the above existence result the martingale problem for $-\widetilde{p}_{x_{0}}(x, D)$ is well-posed.

To proceed with the proof of Theorem 1.2 we choose a sequence $\widetilde{p}_{k}(x, \xi)=\widetilde{p}_{x_{k}}(x, \xi), k \in \mathbb{N}$, out of the family $\left\{\widetilde{p}_{x}(x, \xi)\right\}_{x \in \mathbb{R}^{n}}$ such that $\bigcup_{k \in \mathbb{N}} U_{x_{k}}=\mathbb{R}^{n}$. Since $\widetilde{p}_{k}(x, \xi)$ coincides with $\widetilde{p}(x, \xi)$ for $x \in U_{x_{k}}$, Theorem 5.1 implies that the martingale problem for $-\widetilde{p}(x, D)$ is wellposed.

The statement of Theorem 1.2 for $-p(x, D)$ or equivalently $-\widetilde{p}(x, D)$ is therefore implied by Theorem 5.1 , once we know that $-\widetilde{p}(x, D)$ is an operator in $C_{\infty}\left(\mathbb{R}^{n}\right)$, that is

$$
-\widetilde{p}(x, D): C_{0}^{\infty}\left(\mathbb{R}^{n}\right) \longrightarrow C_{\infty}\left(\mathbb{R}^{n}\right) .
$$

But this follows immediately by [11, Theorem 3.3], since by our assumptions $\sup _{x \in \mathbb{R}^{n}} \widetilde{p}(x, \xi) \longrightarrow 0$ as $\xi \longrightarrow 0$.

Acknowledgements. Financial support by DFG-Habilitanden-Stipendium Ho $1617 / 2-\mathrm{x}$ is gratefully acknowledged.

\section{References.}

[1] Bass, R. F., Uniqueness in law for pure jump Markov processes. Probab. Theor. Rel. Fields 79 (1988), 271-287.

[2] Beauzamy, A., Espaces de Sobolev et Besov d'ordre variable définis sur $L^{p}$. C. R. Acad. Sci. Paris (Ser. A) 274 (1972), 1935-1938.

[3] Berg, C., Forst, G., Potential theory on locally Abelian groups. Ergebnisse der Mathematik und ihrer Grenzgebiete, II. Ser. Bd. 87, Springer Verlag, 1975.

[4] Courrège, Ph., Sur la forme intégro-différentielle des opérateurs de $C_{k}^{\infty}$ dans $C$ satisfaisant au principe du maximum. Sém. Théorie du Potentiel, 1965/66.

[5] Ethier, S. N., Kurtz, Th. G., Markov processes - characterization and convergence. Wiley Series in Probability and Mathematical Statistics, John Wiley, 1986.

[6] Fraenkel, L. E., Formulae for high derivatives of composite functions. Math. Proc. Camb. Phil. Soc. 83 (1978), 159-165. 
[7] Hoh, W., The martingale problem for a class of pseudo differential operators. Math. Ann. 300 (1994), 121-147.

[8] Hoh, W., Pseudo differential operators with negative definite symbols and the martingale problem. Stochastics and Stoch. Rep. 55 (1995), $225-252$.

[9] Hoh, W., A symbolic calculus for pseudo differential operators generating Feller semigroups. Osaka J. Math. 35 (1998), 789-820.

[10] Hoh, W., Un calcul symbolique pour des opérateurs pseudo-différentiels engendrant des semi-groupes de Feller. C. R. Acad. Sci. Paris 325 (1997), 1121-1124.

[11] Hoh, W., On perturbations of pseudo differential operators with negative definite symbol. To appear in Appl. Math. Optim.

[12] Jacob, N., Feller semigroups, Dirichlet forms and pseudo differential operators. Forum Math. 4 (1992), 433-446.

[13] Jacob, N., Further pseudo differential operators generating Feller semigroups and Dirichlet forms. Revista Mat. Iberoamericana 9 (1993), 373-407.

[14] Jacob, N., A class of Feller semigroups generated by pseudo differential operators. Math. Z. 215 (1994), 151-166.

[15] Jacob, N., Leopold, H.-G., Pseudo-differential operators with variable order of differentiation generating Feller semigroups. Integr. Equations Oper. Theor. 17 (1993), 544-553.

[16] Kato, T., Perturbation theory for linear operators. Die Grundlehren der mathematischen Wissenschaften, 132 Springer Verlag, 1966.

[17] Leopold, H.-G., On a class of function spaces and related pseudo-differential operators. Math. Nachr. 127 (1986), 65-82.

[18] Leopold, H.-G., On Besov spaces of variable order of differentiation. $Z$. Anal. Anwendungen 8 (1989), 69-82. Integr. Equations Oper. Theor. 17 (1993), 544-553.

[19] Leopold, H.-G., On function spaces of variable order of differentiation. Forum Math. 3 (1991), 69-82.

[20] Louhivaara, I. S., Simader, C. G., Über koerzitive lineare partielle Differentialoperatoren: Fredholmsche verallgemeinerte Dirichletprobleme und deren Klasseneinteilung. Complex analysis and its applications. Collect. Artic. Steklov Math Inst. (1978), 342-345.

[21] Louhivaara, I. S., Simader, C. G., Fredholmsche verallgemeinerte Dirichletprobleme für koerzitive lineare partielle Differentialoperatoren. Proc. Ralf Nevanlinna Symp. on complex analysis, Silivri, 1976. Publ. Math. Res. Inst. 7 (1978), 47-57. 
[22] Kikuchi, K., Negoro, A., On Markov process generated by pseudodifferential operator of variable order. Osaka J. Math. 34 (1997), 319-335.

[23] Kumano-go, H., Pseudodifferential operators. M.I.T. Press, 1981.

[24] Ma, Zh.-M., Röckner, M., Dirichlet forms. Springer Verlag, 1992.

[25] Negoro, A., Stable-like processes. Construction of the transition density and behavior of sample paths near $t=0$. Osaka J. Math. 31 (1994), 189-214.

[26] Unterberger, A., Sobolev spaces of variable order and problems of convexity for partial differential operators with constant coefficients. Astérique 2/3 (1973), 325-341.

[27] Unterberger, A., Bokobza, J., Sur une généralisation des opérateurs de Calderon-Zygmund et des espaces $H^{s}$. C. R. Acad. Sci. Paris (Ser. A) 260 (1965), 3265-3267.

[28] Unterberger, A., Bokobza, J., Les opérateurs pseudodifférentiels d'ordre variable. C. R. Acad. Sci. Paris (Ser. A) 261 (1965), 2271-2273.

[29] Tsuchiya, M., Lévy measure with generalized polar decomposition and the associated SDE with jumps. Stochastics and Stoch. Rep. 38 (1992), 95-117.

[30] van Casteren, J., On martingales and Feller semigroups. Results. Math. 21 (1992), 274-288.

[30] Višik, M. I., Eskin, G. I., Elliptic convolution equations in a bounded region and their applications. Russian Math. Surveys 22 (1967), 13-75.

[32] Višik, M. I., Eskin, G. I., Convolution equations of variable order. Trans. Moskow Math. Soc. 16 (1968), 27-52.

Recibido: 11 de mayo de 1.998

Walter Hoh

Fakultät für Mathematik

Universität Bielefeld

Postfach 100131

D-33501-Bielefeld, GERMANY

hoh@Mathematik. Uni-Bielefeld.DE 